\title{
Application of the Preview Control Method to the Optimal Tracking Control Problem for Continuous-Time Systems with Time-Delay
}

\author{
Fucheng Liao and Haishan Xu \\ School of Mathematics and Physics, University of Science and Technology Beijing, Beijing 100083, China \\ Correspondence should be addressed to Fucheng Liao; fcliao@ustb.edu.cn
}

Received 10 March 2015; Revised 27 July 2015; Accepted 2 August 2015

Academic Editor: Asier Ibeas

Copyright @ 2015 F. Liao and H. Xu. This is an open access article distributed under the Creative Commons Attribution License, which permits unrestricted use, distribution, and reproduction in any medium, provided the original work is properly cited.

This paper considers the application of the preview control method to the optimal tracking control problem for a class of continuoustime systems with state and input delays. First, through a transformation, the system is transformed into a nondelayed one. Then, the tracking problem of the time-delay system is transformed into one of a nondelayed system via processing of the reference signal. We then apply preview control theory to derive an augmented system for the nondelayed system and design a controller with preview function assuming that the reference signal is previewable. Finally, we obtain the optimal control law of the augmented error system and thus obtain that of the original system by letting the preview length of the reference signal go to zero. Numerical simulations are provided to illustrate the effectiveness and validity of our conclusions.

\section{Introduction}

As we all know, a time-delay phenomenon exists widely in practical engineering, and it is usually the main source of instability and performance degradation in various control systems. Therefore, considerable research has been devoted to study and analysis of time-delay systems. It is a common practice to transform the time-delay system into a nondelayed system in research work of control theory. Actually, discrete time-delay systems can eliminate delay by using the discrete lifting technique [1]. As for continuous timedelay systems, these usually can be converted to nondelayed systems by a transformation. References [2, 3] introduced state-delay systems according to this insight, and in the literature [4-6] they have studied input-delay systems. Reference [7] has discussed systems with state and input delays.

In many industrial applications, optimal tracking control [8-11], as the combination of optimal control and tracking control, aims at looking for an optimal control law to minimize the given performance index function, that is to say, to make the system output track the reference signal in an optimal way. Thus, optimal tracking control has been a goal pursued unremittingly in many fields. Preview control is a control technique to improve the performance of a closedloop system via sufficient utilization of the known future information on the reference signals or disturbances. Preview control theory has attracted academic attention since it was proposed 50 years ago [12-16]. In the existing literature, the most extensive research into preview control centers on the linear quadratic optimal control problem with preview compensation [17-21].

We notice that [7] studied sliding mode control and the regulator problem, but the method of eliminating time-delay is universally applicable. Furthermore, a preview control system will be a normal control system when the preview length goes to zero. Based on these observations, the contribution of this paper is to apply preview control theory to the optimal tracking control problem for continuous-time control systems that are subject to state and input delays. First, according to a transformation in the literature [7], the timedelay system is transformed into the form of a nondelayed system. Second, by dealing with the reference signal, the 
tracking problem of the time-delay system is converted to the tracking problem of the nondelayed system. Then, taking advantage of the methods of preview control theory to derive an augmented error system for the nondelayed system, a controller with preview action for the augmented error system can be obtained, assuming that the reference signal is previewable. Finally, we propose an optimal control law for the augmented error system when the preview length of the reference signal goes to zero and obtain the optimal control law of the original time-delay system. Numerical simulations show the effectiveness and validity of the proposed conclusions in this paper.

The paper is organized as follows. Section 2 proposes the problem and states the basic assumptions. In Section 3, a transformation is introduced that transforms the timedelay system into the form of a nondelayed system. An augmented error system is derived and the optimal controller is presented in Section 4. The existence conditions of the controller are discussed in Section 5. Section 6 provides numerical simulations and Section 7 is the brief conclusion.

\section{Problem Statement and Basic Assumptions}

Consider a continuous-time control system with state and input delays described as

$$
\begin{aligned}
& \dot{x}(t)=A_{0} x(t)+A_{1} x(t-\beta)+B_{1} u(t-\tau), \\
& y(t)=C x(t), \\
& x(\theta)=x_{0}(t), \quad \theta \in[-\beta, 0],
\end{aligned}
$$

where $x(t) \in R^{n}$ is the state vector, $u(t) \in R^{r}$ is the input control vector, $y(t) \in R^{p}$ is the output vector, the matrices $A_{0}, A_{1} \in R^{n \times n}, B_{1} \in R^{n \times r}$, and $C \in R^{p \times n}$ are known real constant matrices with appropriate dimensions, and $\beta, \tau>0$ denotes the constant time-delay, which appears in the state and input vectors, respectively.

We have the following assumptions for system (1).

Assumption 1. $A_{0}$ is invertible.

Assumption 2. The reference signal $y_{d}(t) \in R^{p}$ is a piecewisecontinuously differentiable function.

Assumption 3. The matrix $\left[\begin{array}{cc}A_{0}+M A_{1} & N B_{1} \\ C & 0\end{array}\right]$ is of full row rank; the pair $\left(A_{0}+M A_{1}, N B_{1}\right)$ is stabilizable; the pair $\left(C, A_{0}+\right.$ $\left.M A_{1}\right)$ is detectable. Here,

$$
\begin{aligned}
& M=\left[A_{1} A_{0}^{-1}\left(e^{A_{0} \beta}-I\right)+e^{A_{0} \beta}\right]^{-1}, \\
& N=\left[A_{1} A_{0}^{-1}\left(e^{A_{0} \tau}-I\right)+e^{A_{0} \tau}\right]^{-1} .
\end{aligned}
$$

Define the subtraction of the reference signal $y_{d}(t)$ from the output vector $y(t)$ as the tracking error:

$$
e(t)=y(t)-y_{d}(t)
$$

The objective of this paper is to design a controller to make the output vector $y(t)$ accurately track the reference signal $y_{d}(t)$ without static errors; namely,

$$
\lim _{t \rightarrow \infty} e(t)=0
$$

For this, we introduce the quadratic performance index:

$$
J=\int_{0}^{\infty}\left[e^{T}(t) Q_{e} e(t)+\dot{u}^{T}(t) R \dot{u}(t)\right] d t
$$

where $Q_{e} \in R^{p \times p}$ and $R \in R^{r \times r}$ are both positive definite matrices.

As has been pointed out in [16], introducing the derivative of the input control vector $\dot{u}(t)$ into the quadratic performance index $J$ can create the controller with integral action, which helps the system eliminate static errors.

\section{System Transformation}

First, we eliminate the time-delay of system (1) through a transformation. Utilizing the transformation in the literature [7],

$$
\begin{aligned}
z(t)= & x(t)+\int_{t-\beta}^{t} e^{A(t-\beta-\theta)} A_{1} x(\theta) d \theta \\
& +\int_{t-\tau}^{t} e^{A(t-\theta)} B u(\theta) d \theta
\end{aligned}
$$

where $A, B$ satisfy

$$
\begin{aligned}
& A=A_{0}+e^{-A \beta} A_{1}, \\
& B=e^{-A \tau} B_{1}
\end{aligned}
$$

and the state equation of system (1) is converted to the nondelayed form:

$$
\dot{z}(t)=A z(t)+B u(t)
$$

Now, we solve $A$ and $B$ from (7). Treat $\beta$ as a variable. Left multiply $e^{A \beta}$ on both sides of (7), which by transposition gives

$$
e^{A \beta} A-e^{A \beta} A_{0}=A_{1}
$$

that is,

$$
\frac{d}{d \beta}\left(e^{A \beta}\right)-\left(e^{A \beta}\right) A_{0}=A_{1} .
$$

Then, right multiplying $e^{-A_{0} \beta}$ on both sides, we obtain

$$
\frac{d}{d \beta}\left(e^{A \beta}\right) e^{-A_{0} \beta}+\left(e^{A \beta}\right) \frac{d}{d \beta}\left(e^{-A_{0} \beta}\right)=A_{1} e^{-A_{0} \beta}
$$


namely,

$$
\left[\frac{d}{d \beta}\left(e^{A \beta} e^{-A_{0} \beta}\right)\right]=A_{1} e^{-A_{0} \beta} .
$$

Integrate $[0, \beta]$ on both sides and get

$$
e^{A \beta} e^{-A_{0} \beta}-I=A_{1} \int_{0}^{\beta} e^{-A_{0} \sigma} d \sigma .
$$

Therefore,

$$
e^{A \beta} e^{-A_{0} \beta}-I=A_{1} A_{0}^{-1}\left(I-e^{-A_{0} \beta}\right) .
$$

Thus, we have

$$
e^{A \beta}=A_{1} A_{0}^{-1}\left(e^{A_{0} \beta}-I\right)+e^{A_{0} \beta} .
$$

Then, substituting (15) into the first formula of (7) obtains $A$ :

$$
A=A_{0}+\left[A_{1} A_{0}^{-1}\left(e^{A_{0} \beta}-I\right)+e^{A_{0} \beta}\right]^{-1} A_{1} .
$$

Similarly, substituting (15) into the second formula of (7) gives $B$ :

$$
B=e^{-A \tau} B_{1}=\left[A_{1} A_{0}^{-1}\left(e^{A_{0} \tau}-I\right)+e^{A_{0} \tau}\right]^{-1} B_{1} .
$$

According to $z(t)=x(t)+\int_{t-\beta}^{t} e^{A(t-\beta-\theta)} A_{1} x(\theta) d \theta+$ $\int_{t-\tau}^{t} e^{A(t-\theta)} B u(\theta) d \theta$, we know $z(t)$ is reasonable to be the state vector of (8). Considering the output equation $y(t)=C x(t)$ of system (1) and the relationship of $x(t)$ and $z(t)$, we can take $w(t)=C z(t)$ as the output equation of system (8).

Hence, time-delay system (1) is reduced to the following nondelayed system:

$$
\begin{aligned}
\dot{z}(t) & =A z(t)+B u(t), \\
w(t) & =C z(t) .
\end{aligned}
$$
Let

Then, we make a transformation to the reference signal.

$$
\begin{aligned}
r(t)= & y_{d}(t)+C \int_{t-\beta}^{t} e^{A(t-\beta-\theta)} A_{1} x(\theta) d \theta \\
& +C \int_{t-\tau}^{t} e^{A(t-\theta)} B u(\theta) d \theta .
\end{aligned}
$$

Treating (18) as the control system equation and (19) as the reference signal, we determine that the tracking error of system (1) remains identical with the tracking error of system (18); that is,

$$
e(t)=y(t)-y_{d}(t)=w(t)-r(t) .
$$

Thus, the performance index (5) can be directly applied to system (18). Note that $\int_{t-\beta}^{t} e^{A(t-\beta-\theta)} A_{1} x(\theta) d \theta$ and $\int_{t-\tau}^{t} e^{A(t-\theta)} B u(\theta) d \theta$, as the integrations for historical values of the state vector and the input control vector, respectively, are both known at each instant of time $t$; therefore, $r(t)$ is a known vector. It is shown that taking $r(t)$ as the reference signal of system (18) is reasonable.
Now, the problem is converted to designing the optimal control law of system (18) under performance index (5), where $e(t)=w(t)-r(t)$ is the tracking error.

\section{Augmented Error System and Optimal Control}

At first, the methods of preview control theory are adopted deriving an augmented error system that transforms the tracking problem into a regulator problem.

We derive tracking error $e(t)$ and get

$$
\dot{e}(t)=\dot{w}(t)-\dot{r}(t)=C \dot{z}(t)-\dot{r}(t) .
$$

Differentiating both sides of (8), we have

$$
\frac{d}{d t} \dot{z}(t)=A \dot{z}(t)+B \dot{u}(t) .
$$

Combining (21) and (22) gives

$$
\dot{X}(t)=\widetilde{A} X(t)+\widetilde{B} \dot{u}(t)-\widetilde{D} \dot{r}(t),
$$

where $X(t), \widetilde{A}, \widetilde{B}$, and $\widetilde{D}$ are, respectively, given by

$$
\begin{aligned}
X(t) & =\left[\begin{array}{l}
e(t) \\
\dot{z}(t)
\end{array}\right], \\
\widetilde{A} & =\left[\begin{array}{ll}
0 & C \\
0 & A
\end{array}\right], \\
\widetilde{B} & =\left[\begin{array}{l}
0 \\
B
\end{array}\right], \\
\widetilde{D} & =\left[\begin{array}{l}
I \\
0
\end{array}\right] .
\end{aligned}
$$

According to the output equation of system (18), we now take $e(t)=\left[\begin{array}{ll}I & 0\end{array}\right] X(t)$ as the output equation and get

$$
\begin{aligned}
\dot{X}(t) & =\widetilde{A} X(t)+\widetilde{B} \dot{u}(t)-\widetilde{D} \dot{r}(t), \\
e(t) & =\widetilde{C} X(t),
\end{aligned}
$$

where $\widetilde{C}=\left[\begin{array}{ll}I & 0\end{array}\right] \in R^{p+n}$.

To utilize the research results of preview control, we assume that the reference signal $r(t)$ is previewable and the preview length is $l_{r}$. After obtaining the control input and letting $l_{r} \rightarrow 0^{+}$, we can obtain the solution of the problem in this paper.

Equations (25) are the needed augmented error system. Our basic ideas are as follows: applying the idea of optimal preview control, designing the controller of system (25), and then obtaining the controller of system (1). It is easy to see that if we can design a state feedback to make the closedloop system of system (25) asymptotically stable, we will have $\lim _{t \rightarrow \infty} X(t)=0$. Hence, the error signal $e(t)$ as a partial component of the state vector $X(t)$ will satisfy (4). 
$y_{d}(t)$ is a piecewise-continuously differentiable function; so $r(t)$ is piecewise-continuously differentiable.

According to the state and input vectors of (25), the performance index function (5) can be denoted as follows:

$$
J=\int_{0}^{\infty}\left[X^{T}(t) \widetilde{Q} X(t)+\dot{u}^{T}(t) R \dot{u}(t)\right] d t
$$

where $\widetilde{Q}=\widetilde{C}^{T} Q_{e} \widetilde{C}=\left[\begin{array}{cc}Q_{e} & 0 \\ 0 & 0\end{array}\right]$.

Meanwhile, noting that the form of system (25) and the performance index function are similar to those mentioned in the literature [22], we obtain the following theorem by using similar derivations of [22].

Theorem 4. Suppose $(\widetilde{A}, \widetilde{B})$ is stabilizable and $\left(\widetilde{Q}^{1 / 2}, \widetilde{A}\right)$ is detectable. The optimal control input of system (25) to minimize performance index function (26) is given by

$$
\dot{u}(t)=-R^{-1} \widetilde{B}^{T} P X(t),
$$

where

$$
\widetilde{A}_{c}=\widetilde{A}-\widetilde{B} R^{-1} \widetilde{B}^{T} P
$$

and $P$ is a positive semidefinite matrix satisfying the algebraic Riccati equation

$$
\widetilde{A}^{T} P+P \widetilde{A}-P \widetilde{B} R^{-1} \widetilde{B}^{T} P+\widetilde{Q}=0
$$

Proof. According to the results of $[22]$, when $(\widetilde{A}, \widetilde{B})$ is stabilizable, $\left(\widetilde{Q}^{1 / 2}, \widetilde{A}\right)$ is detectable, and $l_{r}$ is the preview length of the reference signal $r(t)$, the optimal preview control input of system (25) is

$$
\dot{u}(t)=-R^{-1} \widetilde{B}^{T} P X(t)+R^{-1} \widetilde{B}^{T} g\left(t, l_{r}\right),
$$

where

$$
g\left(t, l_{r}\right)=\int_{t}^{t+l_{r}} \exp \left[\widetilde{A}_{c}^{T}(s-t)\right] P \widetilde{D} \dot{r}(s) d s
$$

is the desired output preview compensation of system (25) and $\widetilde{A}_{c}$ and $P$ are described by (28) and (29), respectively.

Because $r(t)$ is not previewable, $\dot{r}(t)$ is not previewable. Therefore, $g\left(t, l_{r}\right)$ is unsolvable. To overcome the difficulty, we let $l_{r} \rightarrow 0^{+}$utilize the property of integration and get

$$
\lim _{l_{r} \rightarrow 0^{+}} g\left(t, l_{r}\right)=0
$$

Making $l_{r} \rightarrow 0^{+}$on both sides of (30), we give (27) immediately because $g\left(t, l_{r}\right)$ is only related to $l_{r}$. The proof is complete.

Returning to system (1), we get the following.
Theorem 5. Suppose $(\widetilde{A}, \widetilde{B})$ is stabilizable and $\left(\widetilde{Q}^{1 / 2}, \widetilde{A}\right)$ is detectable. Let $x(t)=0$, [namely, $x_{0}(t) \equiv 0$ in system (1)] $u(t)=0$, and $y_{d}(t)=0$, for $t \leq 0$; the optimal control input of system (1) to minimize performance index (5) is given by

$$
\begin{gathered}
u(t)=-K_{e} \int_{0}^{t} e(\sigma) d \sigma-K_{x}[x(t) \\
+\int_{t-\beta}^{t} e^{A(t-\beta-\theta)} A_{1} x(\theta) d \theta \\
\left.+\int_{t-\tau}^{t} e^{A(t-\theta)} B u(\theta) d \theta\right]
\end{gathered}
$$

where $P$ is the unique positive semidefinite solution of Riccati equation (29), $K_{e}=R^{-1} \widetilde{B}^{T} P_{e}, K_{x}=R^{-1} \widetilde{B}^{T} P_{x}$, and $P=$ $\left[\begin{array}{ll}P_{e} & P_{x}\end{array}\right]$

The method of proof is similar to that in [22] and is omitted here.

\section{Existence Conditions of the Controller}

In this section, we discuss conditions of the original system (1) that can make $(\widetilde{A}, \widetilde{B})$ stabilizable and $\left(\widetilde{Q}^{1 / 2}, \widetilde{A}\right)$ detectable.

Lemma 6. The pair $(\widetilde{A}, \widetilde{B})$ is stabilizable if and only if $(A, B)$ is stabilizable and the matrix $\left[\begin{array}{ll}A & B \\ C & 0\end{array}\right]$ is of full row rank.

Lemma 7. The pair $\left(\widetilde{Q}^{1 / 2}, \widetilde{A}\right)$ is detectable if and only if $(C, A)$ is detectable.

Lemmas 6 and 7 are both the results of [22].

Note that we can put assumption 3 another way: suppose the matrix $\left[\begin{array}{ll}A & B \\ C & 0\end{array}\right]$ is of full row rank, the pair $(A, B)$ is stabilizable, and $(C, A)$ is detectable.

Note the following. As seen from Lemmas 6 and 7, when $\left(A_{0}, B_{1}\right)$ or $\left(A_{1}, B_{1}\right)$ is stabilizable, this cannot guarantee the stabilizability of $(\widetilde{A}, \widetilde{B})$; similarly, when $\left(C, A_{0}\right)$ or $\left(C, A_{1}\right)$ is detectable, we cannot guarantee the detectability of $\left(\widetilde{Q}^{1 / 2}, \widetilde{A}\right)$. These are characteristics of time-delay systems.

In summary, we obtain the main theorem in this paper.

Theorem 8. Suppose the following conditions are satisfied:

(1) $A_{0}$ is invertible (Assumption 1).

(2) The reference signal $y_{d}(t)$ is a piecewise-continuously differentiable function (Assumption 2).

(3) The matrix $\left[\begin{array}{cc}A_{0}+M A_{1} & N B_{1} \\ C & 0\end{array}\right]$ is of full row rank, the pair $\left(A_{0}+M A_{1}, N B_{1}\right)$ is stabilizable, and the pair $\left(C, A_{0}+\right.$ $M A_{1}$ ) is detectable (Assumption 3).

(4) $Q_{e}$ and $R$ are both positive definite matrices. 
We then have the following closed-loop system:

$$
\begin{aligned}
\dot{x}(t)= & A_{0} x(t)+A_{1} x(t-\beta)+B_{1} u(t-\tau), \\
y(t)= & C x(t) \\
u(t)= & -K_{e} \int_{0}^{t} e(\sigma) d \sigma-K_{x} x(t) \\
& -K_{x} \int_{t-\beta}^{t} e^{A(t-\beta-\theta)} A_{1} x(\theta) d \theta \\
& -K_{x} \int_{t-\tau}^{t} e^{A(t-\theta)} B u(\theta) d \theta,
\end{aligned}
$$

where $K_{e}, K_{x}$, and $P$ are all defined in Theorem 5 and the output vector $y(t)$ of the closed-loop system can track the reference signal $y_{d}(t)$ asymptotically without static errors.

\section{Numerical Simulation}

Consider the system

$$
\begin{aligned}
\dot{x}(t)= & {\left[\begin{array}{cc}
0 & 1 \\
-1 & 1
\end{array}\right] x(t)+\left[\begin{array}{ll}
0 & 1 \\
1 & 1
\end{array}\right] x(t-\beta) } \\
& +\left[\begin{array}{l}
1 \\
2
\end{array}\right] u(t-\tau), \\
y(t)= & {\left[\begin{array}{ll}
1 & 0
\end{array}\right] x(t) . }
\end{aligned}
$$

In comparison with system (1), the coefficient matrices are as follows:

$$
\begin{aligned}
A_{0} & =\left[\begin{array}{cc}
0 & 1 \\
-1 & 1
\end{array}\right], \\
A_{1} & =\left[\begin{array}{ll}
0 & 1 \\
1 & 1
\end{array}\right], \\
B_{1} & =\left[\begin{array}{l}
1 \\
2
\end{array}\right], \\
C & =\left[\begin{array}{ll}
1 & 0
\end{array}\right] .
\end{aligned}
$$

Taking the sampling period $T=0.01 \mathrm{~s}$ and using the discretization technique for (35), we obtain the following discrete-time system:

$$
\begin{aligned}
x((k+1) T)= & \left(T A_{0}+I\right) x(k T)+T A_{1} x(k T-\beta) \\
& +T B_{1} u(k T-\tau), \\
y(k T)= & C x(k T) .
\end{aligned}
$$

According to Theorem 8, we get the closed-loop system:

$$
\begin{aligned}
& x((k+1) T)=\left(T A_{0}+I\right) x(k T)+T A_{1} x(k T-\beta) \\
& +T B_{1} u(k T-\tau), \\
& y(k T)=C x(k T), \\
& e(k T)=y(k T)-y_{d}(k T), \\
& u(k T)=-K_{e} \sum_{i=0}^{k-1} T e(i T)-K_{x} x(k T)-K_{x} \\
& \quad \sum_{i=k-\beta / T}^{k-1} T e^{A(k T-\beta-i T)} A_{1} x(i T)-K_{x} \\
& \quad \sum_{i=k-\tau / T}^{k-1} T e^{A(k T-i T)} B u(i T) .
\end{aligned}
$$

Take the step signal below as the reference signal:

$$
y_{d}(t)= \begin{cases}0, & t \leq 3 \\ 1, & t>3\end{cases}
$$

and the weight matrices $Q_{e}=1, R=50$. Now we do numerical simulations for several different time-delay combinations.

(1) The state delay is $\beta=0.30$, and the input delays are $\tau=0, \tau=0.15$, and $\tau=0.30$, respectively. Here, $\tau=0$ means that the system has no input delay. In each case, $A$ and $B$ separately are

$$
\begin{gathered}
\beta=0.30, \tau=0: \\
A=\left[\begin{array}{ll}
-0.43342315236725 & 1.62880145390177 \\
-0.43342315236725 & 1.62880145390177
\end{array}\right], \\
B=\left[\begin{array}{l}
1 \\
2
\end{array}\right],
\end{gathered}
$$

$$
\beta=0.30, \tau=0.15
$$

$$
\begin{aligned}
& A=\left[\begin{array}{ll}
-0.43342315236725 & 1.62880145390177 \\
-0.43342315236725 & 1.62880145390177
\end{array}\right], \\
& B=\left[\begin{array}{l}
0.52165561961054 \\
1.52165561961054
\end{array}\right],
\end{aligned}
$$




$$
\begin{aligned}
& \beta=0.30, \tau=0.30: \\
& A=\left[\begin{array}{ll}
-0.43342315236725 & 1.62880145390177 \\
-0.43342315236725 & 1.62880145390177
\end{array}\right], \\
& B=\left[\begin{array}{l}
0.19537830153452 \\
1.19537830153452
\end{array}\right] \text {. } \\
& \beta=0.30, \tau=0: \\
& P=\left[\begin{array}{ccc}
3.65168053633590 & 5.97598941968832 & 2.59826158915244 \\
5.97598941968832 & 21.42986989326477 & -7.64679690172430 \\
2.59826158915244 & -7.64679690172430 & 67.51973067284588
\end{array}\right], \\
& K_{e}=0.14142135623731 \text {, } \\
& K_{x}=\left[\begin{array}{ll}
-0.00913558840370 & 1.97505566074242
\end{array}\right] \text {, }
\end{aligned}
$$

It is verified that $(A, B)$ is stabilizable and $(C, A)$ is detectable in any case. Together with the reference signal $y_{d}(t)$, which is piecewise-continuously differentiable, the

In each case, we can get $\widetilde{A}, \widetilde{B}, \widetilde{D}$, and $\widetilde{C}$ (omitted here) and have the solutions of Riccati equations, $K_{e}$ and $K_{x}$ :

$$
\beta=0.30, \tau=0.30
$$

\section{$P$}

$$
\begin{aligned}
= & {\left[\begin{array}{lll}
3.839072604513 & 6.058678257824 & 4.925079815110 \\
6.058678257824 & 22.054985350715 & -9.056007357195 \\
4.925079815110 & -9.056007357195 & 107.307706538711
\end{array}\right], } \\
K_{e} & =0.14142135623731, \\
K_{x} & =\left[\begin{array}{lll}
-0.13032578230273 & 2.53007913295354
\end{array}\right] .
\end{aligned}
$$

Take the allowed initial state to be $x(0)=\left[\begin{array}{l}0 \\ 0\end{array}\right]$ and define $x(t)=\left[\begin{array}{l}0 \\ 0\end{array}\right]$ and $u(t)=0$ when $t<0$.

Figure 1 shows the situation in which the output response of the closed-loop system tracks the reference signal. As the input delay $\tau$ decreases, the effect of tracking improves when the state delay $\beta$ is fixed. The control input curves of the closed-loop system are shown in Figure 2, in each case.

(2) The input delay is $\tau=0.20$, and the state delays are $\beta=0.01, \beta=0.15$, and $\beta=0.30$, respectively. Figure 3 shows the situation in which the output response of the closedloop system tracks the reference signal. As the state delay $\beta$ decreases, the effect of tracking improves when the input delay $\tau$ is fixed. The control input curves of the closed-loop system are shown in Figure 4, in each case.

\section{Conclusions}

In this paper, we have studied the optimal tracking control for a class of linear continuous-time control systems with state and input delays. The main contribution of this paper is applying the methods of preview control to the optimal tracking control of linear continuous time-delay control systems. First, the time-delay system is converted to a nondelayed system in form, by a transformation. Second, utilizing the methods of preview control theory to derive an augmented system for the nondelayed system, we can design a controller with preview function, assuming that the reference signal is previewable. Finally, we propose an optimal control law of the augmented error system when the preview length of the reference signal goes to zero and obtain the optimal control law of the original time-delay system. Numerical simulations 


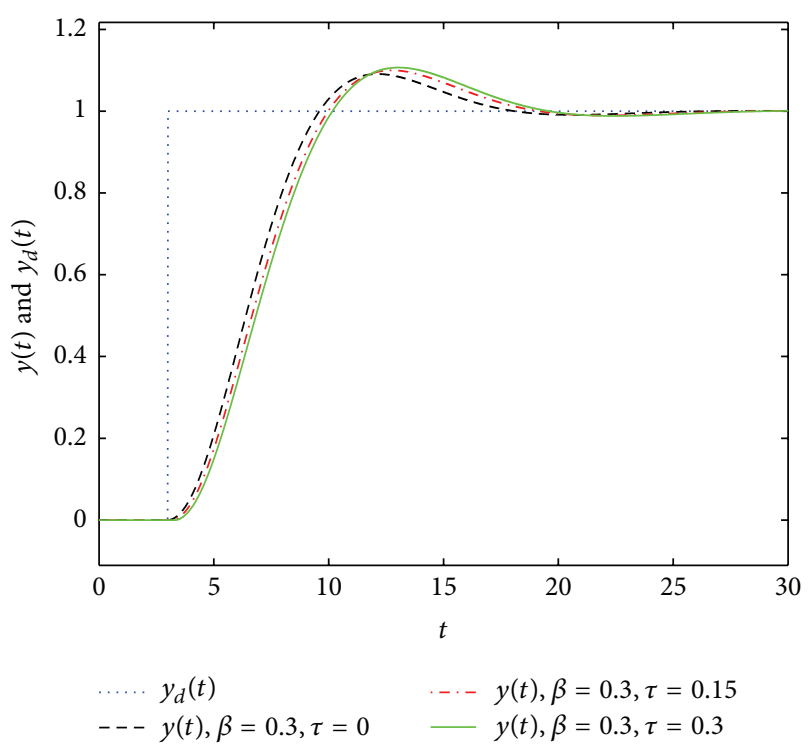

FIGURE 1: Closed-loop responses for different input delays with a fixed state delay.

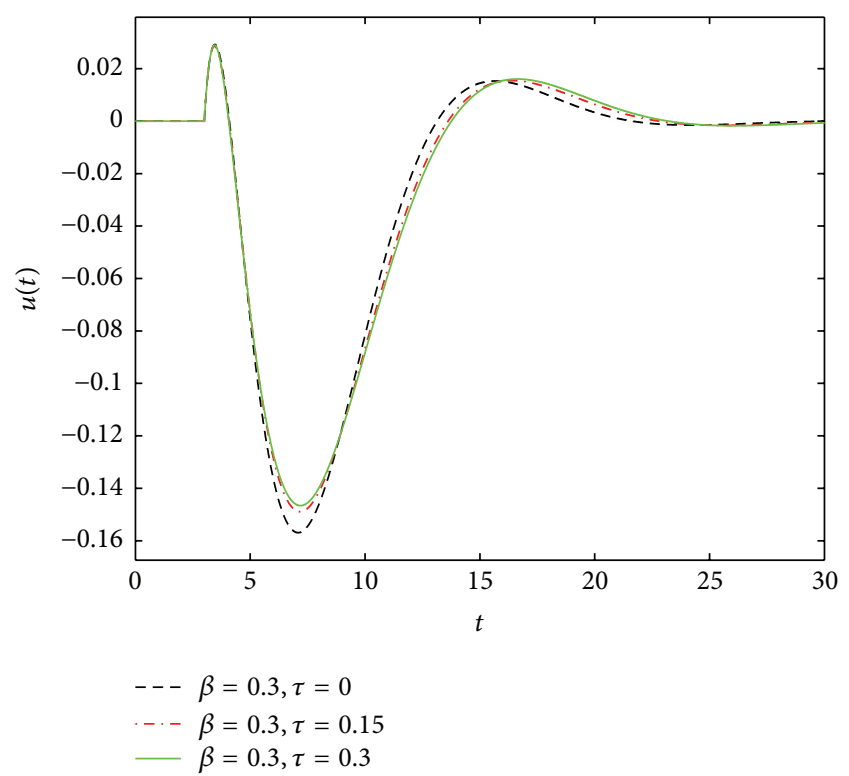

FIGURE 2: The control inputs for different input delays with a fixed state delay.

show the effectiveness and validity of the conclusions in this paper.

\section{Conflict of Interests}

The authors declare that there is no conflict of interests regarding the publication of this paper.

\section{Acknowledgment}

This work is supported by the National Natural Science Foundation of China (no. 61174209).

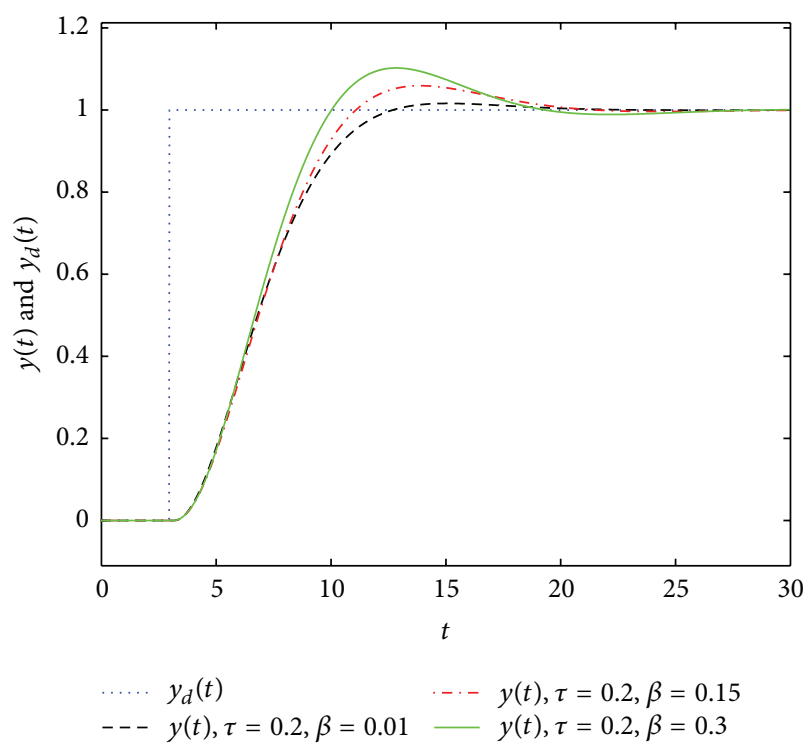

FIGURE 3: Closed-loop responses for different state delays with a fixed input delay.

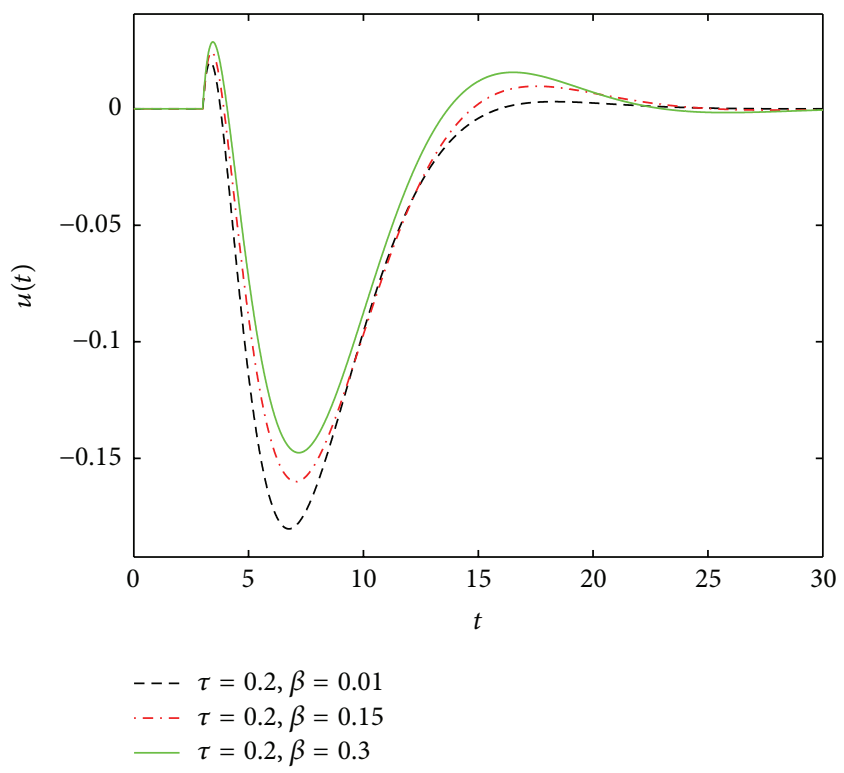

FIgURE 4: The control inputs for different state delays with a fixed input delay.

\section{References}

[1] J. Chu, X. Hu, E. Zhong, and H. Chen, "Optimal tracking control of discrete time-delay systems and its application," Acta Automatica Sinica, vol. 21, no. 1, pp. 25-32, 1995.

[2] X. Lv and J. Li, "Observer-based fault diagnosis and diagnosability for systems with state delays," Journal of Qingdao Agriculture University (Natural Science), vol. 26, no. 3, pp. 233-238, 2009.

[3] J. Li and X. Lv, "Fault diagnosis and diagnosability for linear systems with two kinds of time-delays," Application Research of Computers, vol. 26, no. 10, pp. 3727-3731, 2009.

[4] G.-Y. Tang, J. Lei, and L. Sun, "Observer-based optimal disturbance-rejection for linear systems with time-delay in control 
action," Control Theory and Applications, vol. 26, no. 2, pp. 209214, 2009.

[5] J. Li, R.-H. Ye, and G.-Y. Tang, "Real-time fault diagnosis and optimal fault-tolerant control for systems with control timedelay," Control and Decision, vol. 23, no. 4, pp. 439-443, 2008.

[6] J. Li, R. Ye, and S. Shang, "Fault diagnosis and fault-tolerant control for remote monitoring systems with delayed controls and measurements," Transactions of the Chinese Society of Agricultural Engineering, vol. 24, no. 11, pp. 145-149, 2008.

[7] H. Pang, C. Lin, K. Zhuang, and J. Liu, "Optimal sliding mode control for linear systems with input and state delays," Journal of System Simulation, vol. 18, no. 2, pp. 727-730, 2006.

[8] C.-M. Zhang, G.-Y. Tang, and M. Bai, "Observer-based optimal tracking control for linear systems with control delay," Electric Machines and Control, vol. 11, no. 3, pp. 271-281, 2007.

[9] W.-C. Liu, N.-P. Hu, and G.-L. Li, "Optimal tracking control for time-delay systems with persistent disturbances," Journal of System Simulation, vol. 18, no. 5, pp. 1275-1277, 2006.

[10] Y. Zhao and S. Ge, "Optimal output tracking control for bilinear time-delay systems," Microcomputer and Its Applications, vol. 29, no. 7, pp. 75-78, 2010.

[11] R. Tang, H. Ma, S. Guo, and L. Ren, "Optimal tracking control for linear time-delay large-scale systems with persistent disturbances," Journal of Systems Engineering and Electronics, vol. 20, no. 5, pp. 1058-1064, 2009.

[12] T. Sheridan, “Three models of preview control," IEEE Transactions on Human Factors in Electronics, vol. 7, no. 2, pp. 91-102, 1966.

[13] N. Birla and A. Swarup, "Optimal preview control: a review," Optimal Control Applications and Methods, vol. 36, no. 2, pp. 241-268, 2015.

[14] M. Tomizuka and D. E. Rosenthal, "On the optimal digital state vector feedback controller with integral and preview actions," Journal of Dynamic Systems, Measurement and Control, vol. 101, no. 2, pp. 172-178, 1979.

[15] T. Katayama, T. Ohki, T. Inoue, and T. Kato, "Design of an optimal controller for a discrete-time system subject to previewable demand," International Journal of Control, vol. 41, no. 3, pp. 677-699, 1985.

[16] T. Katayama and T. Hirono, "Design of an optimal servomechan ism with preview action and its dual problem," International Journal of Control, vol. 45, no. 2, pp. 407-420, 1987.

[17] T. Egami and T. Tsuchiya, "Disturbance suppression control with preview action of linear DC brushless motor," IEEE Transactions on Industrial Electronics, vol. 42, no. 5, pp. 494500, 1995.

[18] Q. Zou, "Optimal preview-based stable-inversion for output tracking of nonminimum-phase linear systems," Automatica, vol. 45, no. 1, pp. 230-237, 2009.

[19] S.-H. Kim and H. Yamato, "On the design of a longitudinal motion control system of a fully-submerged hydrofoil craft based on the optimal preview servo system," Ocean Engineering, vol. 31, no. 13, pp. 1637-1653, 2004.

[20] Y.-J. Xu and F.-C. Liao, "Preview control for a class of timevarying discrete systems with input time-delay," Control and Decision, vol. 28, no. 3, pp. 466-470, 2013.

[21] S. Yim, "Design of a preview controller for vehicle rollover prevention," IEEE Transactions on Vehicular Technology, vol. 60, no. 9, pp. 4217-4226, 2011.

[22] F. Liao, Y. Y. Tang, H. Liu, and Y. Wang, "Design of an optimal preview controller for continuous-time systems," International
Journal of Wavelets, Multiresolution and Information Processing, vol. 9, no. 4, pp. 655-673, 2011. 


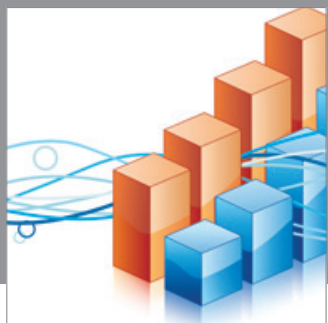

Advances in

Operations Research

mansans

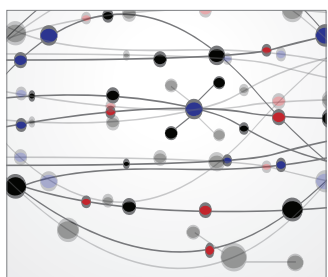

The Scientific World Journal
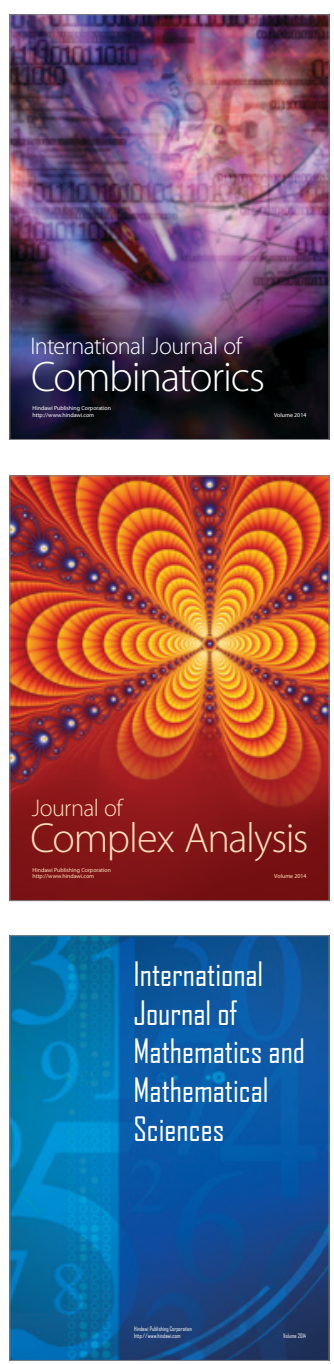
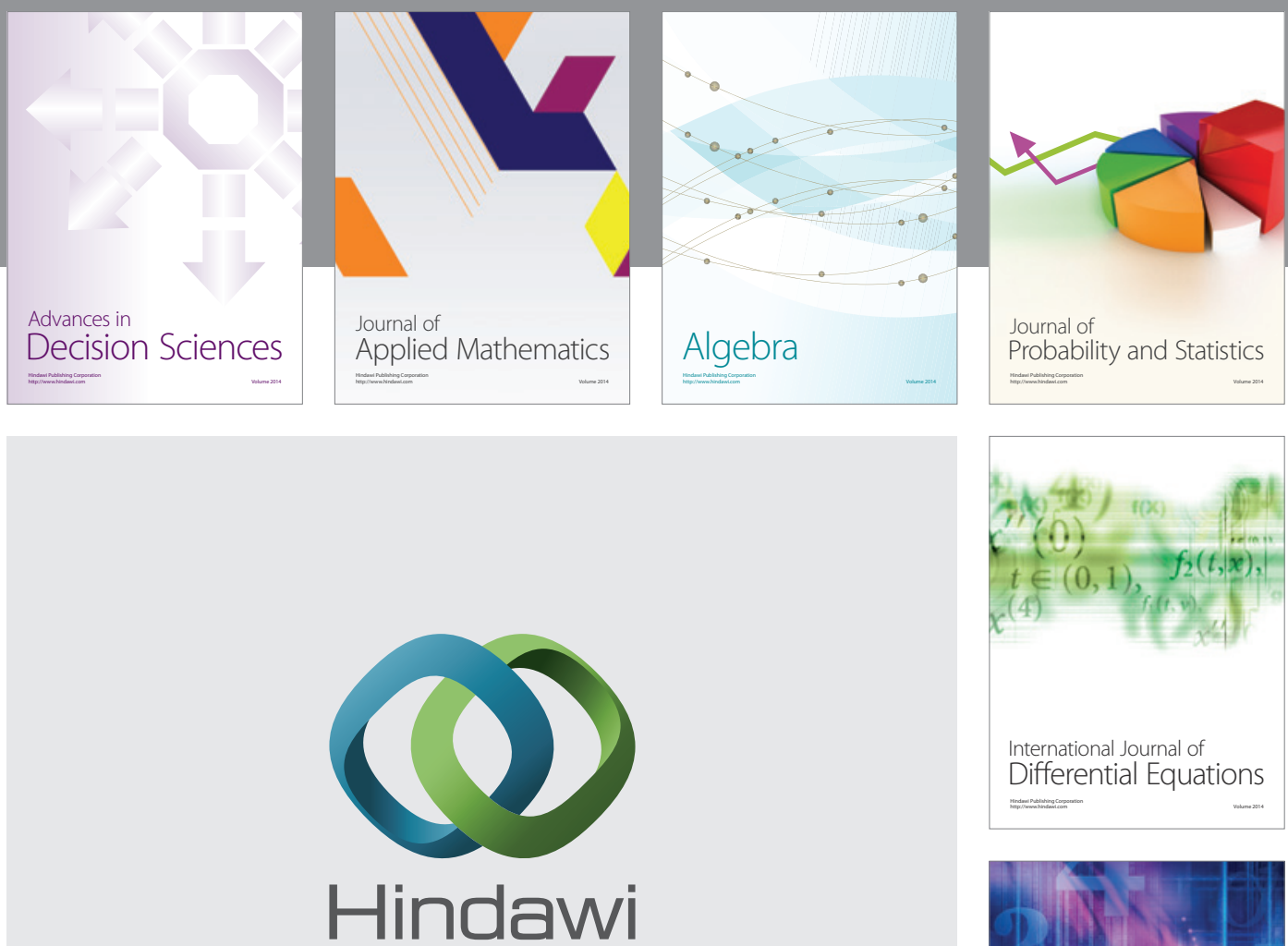

Submit your manuscripts at http://www.hindawi.com
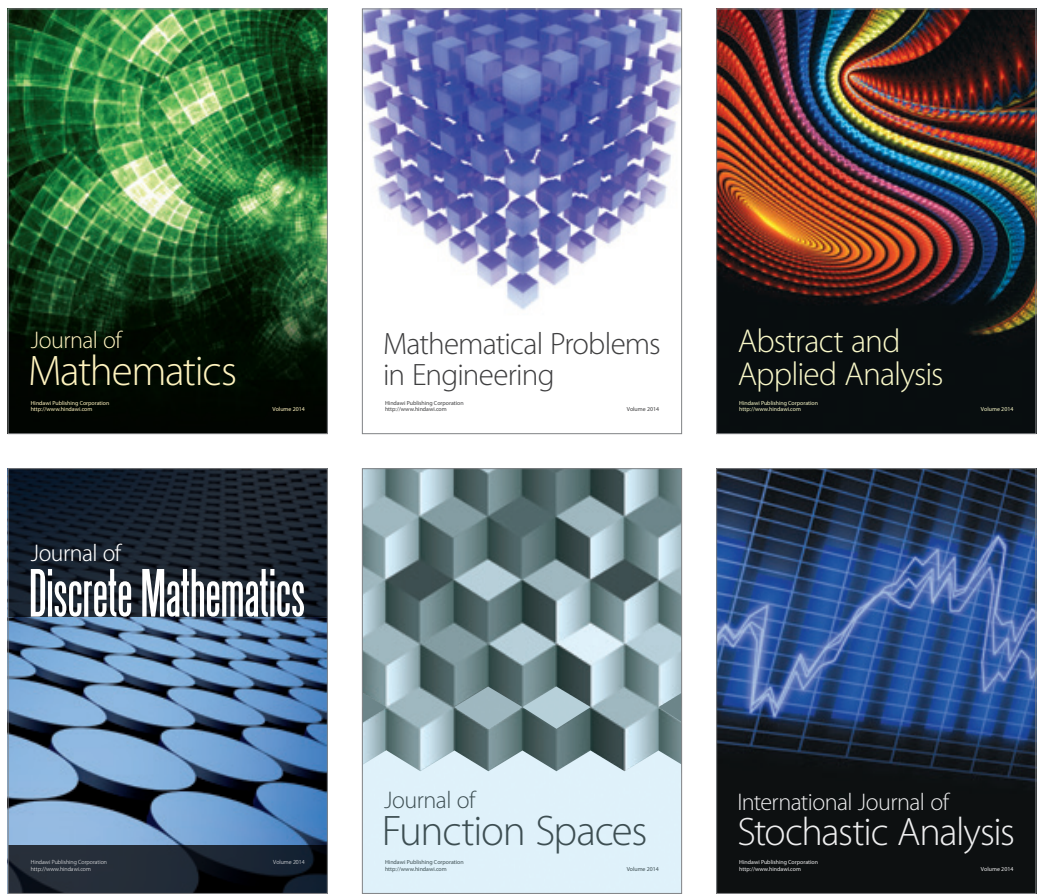

Journal of

Function Spaces

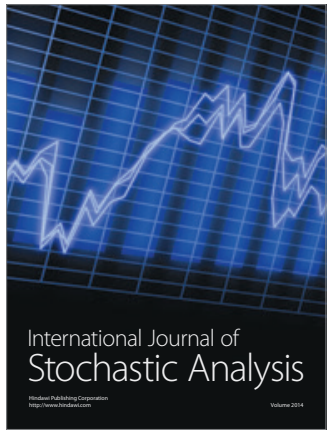

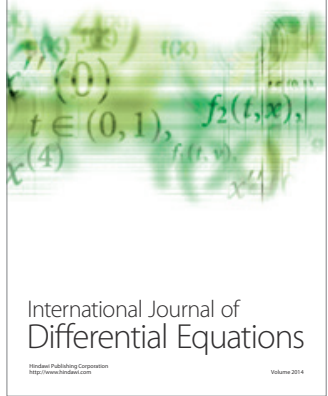
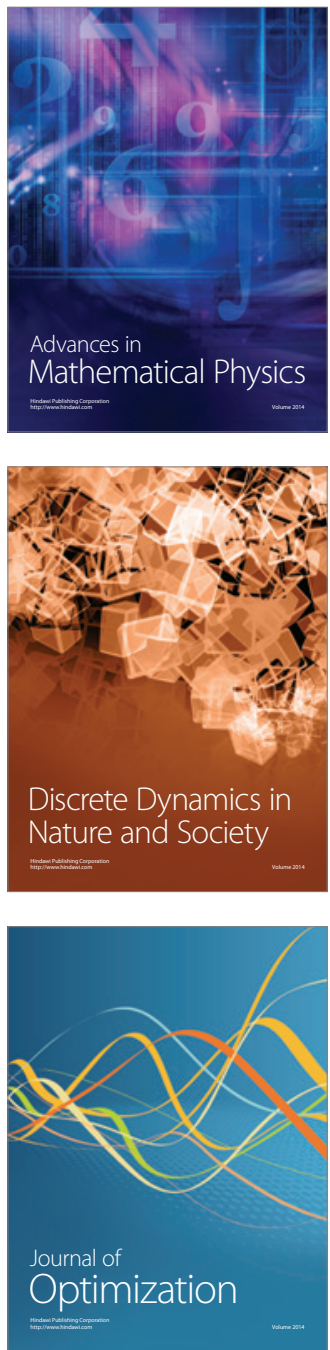